\title{
TropicALL study: Thromboprophylaxis in Children treated for Acute Lymphoblastic Leukemia with Low-molecular-weight heparin: a multicenter randomized controlled trial
}

Irene L. M. Klaassen ${ }^{1,2}$, Mandy N. Lauw ${ }^{2,3}$, Marianne D. van de Wetering ${ }^{4}$, Bart J. Biemond ${ }^{3}$, Saskia Middeldorp², Floor C. H. Abbink ${ }^{5}$, Marc Bierings ${ }^{6}$, D. Maroeska M. W. te Loo ${ }^{7}$, Rob Pieters ${ }^{8}$, Inge M. van der Sluis ${ }^{9}$, Wim J. E. Tissing ${ }^{10}$, C. Michel Zwaan ${ }^{9}$ and C. Heleen van Ommen ${ }^{*}$

\begin{abstract}
Background: Venous thromboembolism (VTE) is a common and severe complication during treatment of acute lymphoblastic leukemia (ALL). An important cause is the intensive use of asparaginase. Prospective cohort studies in which prophylactic low-molecular-weight heparin $(\mathrm{LMWH})$ was used to prevent VTE showed lower VTE risk than in historic control cohorts, with a negligible bleeding risk. However, the efficacy of thromboprophylaxis with LMWH during ALL treatment has never been investigated in a randomized design. Here, we present the protocol of a randomized controlled trial in which the efficacy and safety of thromboprophylaxis with high prophylactic dose LMWH versus no thromboprophylaxis will be assessed in children treated for primary ALL with asparaginase.

Methods/Design: Thromboprophylaxis in Children treated for Acute Lymphoblastic Leukemia with Low-molecularweight heparin (TropicALL) is a multicenter, randomized controlled open-label trial conducted in the Netherlands. Patients between 1 and 19 years of age with primary ALL, who are treated within the Dutch Childhood Oncology Group (DCOG) ALL-11 or 12 study will be randomized to thromboprophylaxis with LMWH once daily, (dose of $85 \mathrm{IU} / \mathrm{kg}$ (intervention $\operatorname{arm} A$ )), or to no thromboprophylaxis (arm B, standard of care) during asparaginase courses of ALL treatment. Primary efficacy endpoint is symptomatic objectified VTE during ALL treatment; secondary efficacy endpoints are overall survival and the composite of symptomatic and asymptomatic objectified VTE. Primary safety endpoints are major bleeding, clinically relevant non-major bleeding and minor bleeding. A total of 324 patients will be included to obtain a relative risk reduction of $75 \%$ with a power of $80 \%$, using a two-sided test with significance level $a=0.05$.
\end{abstract}

Discussion: This trial will be the first to assess efficacy and safety of thromboprophylaxis with LMWH during asparaginase treatment for ALL in children in a randomized design.

Trail registration: Nederlands Trial Register NTR4707. Registered 30 July 2014.

Keywords: Acute lymphoblastic leukemia, Low molecular weight heparin, Venous thromboembolic disease, Asparaginase, Pediatric

\footnotetext{
* Correspondence: c.vanommen@erasmusmc.nl

${ }^{9}$ Department of Pediatric Oncology/Hematology, Erasmus MC-Sophia

Children's Hospital, Rotterdam, The Netherlands

Full list of author information is available at the end of the article
} 


\section{Background}

Acute lymphoblastic leukemia (ALL) is the most common type of childhood cancer, representing a quarter of all pediatric malignancies $[1,2]$. Survival rates for childhood ALL have improved significantly over the past decades, with a current 5-year survival of $86 \%$ [3, 4]. This is a result of substantial improvements in ALL treatment with risk-based therapy, increased treatment intensity and improved supportive care $[5,6]$.

Venous thromboembolism (VTE) is a frequent and severe complication during ALL treatment. Reported incidences of VTE during ALL treatment vary from 1 to $37 \%$ [7]. In unpublished data of the most recent Dutch Childhood Oncology Group (DCOG) ALL study, the ALL-10, incidence of symptomatic VTE was about 10\% [3], but may have been underreported as VTE events were not systematically registered. The majority of VTE during ALL treatment are cerebral sinovenous thrombosis (CSVT) and catheter-related deep-vein thrombosis (DVT) (35-43\%) [710]. CSVT can be severe and life-threatening [7, 8]. Reported overall mortality of CSVT varies between 10 and $21 \%$, however limited data are available $[7,11]$. Long term complications are common; more than $50 \%$ of patients with CSVT suffer from persistent neurological or cognitive impairments, and one fifth of patients has a reported poor quality of life [12-15]. Although mortality is rare in other types VTE such as (catheter-related) DVT, morbidity is frequent with for instance post-thrombotic syndrome (PTS), characterized by swelling, ulceration, pain and dilated collateral veins of the affected limb due to hampered venous flow, being frequently seen, with reported in up to $68 \%$ of patients [16-19].

Risk factors for VTE in ALL patients are well described but difficult to avoid. In particular, asparaginase therapy, which is an important component of ALL therapy, is considered to be a major risk factor for VTE as it reduces levels of natural anticoagulant proteins. Concomitant administration of corticosteroids may amplify this effect $[7,8,20,21]$. In addition, a procoagulant state at diagnosis, presence of central venous catheters (CVCs), inherited prothrombotic defects and infections contribute to the risk of VTE [7, 8, 20, 22].

VTE occurrence leads to suboptimal treatment of ALL patients. In $68 \%$ of CSVT patients, therapy adjustments are necessary [11], which may lead to reduced survival rates [4]. In addition, CVC-related DVT is associated with recurrent catheter complications, such as obstruction of the catheter and catheter-related infections [23].

Currently, there are no evidence-based strategies to prevent VTE complications during ALL treatment. Administration of fresh frozen plasma (FFP) or antithrombin (AT) do not reduce the risk of VTE [24]. A few prospective cohort studies have been published which use low-molecular-weight heparin (LMWH) for thromboprophylaxis during ALL treatment. Elhasid et al. reported on 41 children with ALL who received LMWH prophylaxis during 4 to 8 courses in1824 days of L-asparaginase treatment. During 76 courses of asparaginase, none of the children developed VTE and no bleeding episodes occurred [25]. Mitchell et al. described 19 children with ALL and increased risk for thrombosis, based on a VTE-risk score, including asparaginase, steroids, presence of CVC and thrombophilia. VTE developed in 1 of 8 children who were given prophylactic dose LMWH and in 8 of 11 children without thromboprophylaxis. No bleeding events occurred [26].

However, thromboprophylaxis with LMWH during ALL treatment has not been studied in a randomized design. As the prospective cohort studies showed promising results with a negligible bleeding risk, a large randomized controlled trial is needed to confirm these findings.

\section{Methods \\ Aim and study design}

The TropicALL study is a multicenter randomized controlled open-label trial conducted in the Netherlands. The aim of this study is to assess the efficacy and safety of thromboprophylaxis with LMWH in children treated for primary ALL in the DCOG ALL-11 or subsequent study. As a secondary objective, clinical risk factors will be evaluated to increase insight in the pathogenesis of VTE during ALL treatment and to establish a risk model for these complications. In addition, since a thrombophilic state is associated with a higher VTE risk, specific coagulation assays, including antithrombin, fibrinogen, D-dimer, thrombin antithrombin complex (TAT) and thrombin generation, and genetic thrombophilic mutations Factor $\mathrm{V}$ Leiden and prothrombin G20210A will be performed in patients with and without VTE. The inclusion period is 3 years with a follow-up of 3 months after the end of the ALL treatment period, or until recurrence of ALL, or until death. Primary efficacy endpoint is the incidence of symptomatic objectified VTE during ALL treatment; secondary efficacy endpoints are incidence of the composite of symptomatic and asymptomatic objectified VTE, and the value of plasma coagulation assays to predict the risk of VTE. Primary safety endpoint is major bleeding. Secondary safety endpoints are the incidence of clinically relevant non-major bleeding and minor bleeding, the burden of LMWH injections, and adverse skin reactions.

Patients will be closely monitored for adverse events during the study. All serious adverse events and unexpected adverse reactions, will be reported through the web portal ToetsingOnline to the accredited Ethics Committee that approved the study protocol. Furthermore a Data Monitoring Committee will semi-annually 
review all incidences of adverse events, symptomatic VTE and bleeding complications. To enhance safety of the study, DMC will provide the core committee with recommendations related to the protection of the patients' safety, including stopping recruitment and study treatment.

\section{Definitions}

Symptomatic VTE is defined as suspicion of VTE based on clinical symptoms, for instance for (CVC-associated) DVT: peripheral deep vein thrombosis, swelling, erythema, skin discoloration, increased warmth, pain, tenderness, venous distension, or presence of subcutaneous collateral veins and objectively confirmed by imaging tests. Other forms of VTE (i.e. pulmonary emboli and CSVT) are defined as symptomatic venous thrombosis in any component of the venous or pulmonary arterial circulations or the heart, or the cerebral sinovenous system, requiring therapeutic anticoagulation, acute intervention, life-saving measures, ALL treatment adjustments, or of fatal nature, and objectively confirmed by routine imaging tests. Screening for asymptomatic VTE should not be performed. However, if thrombi are incidentally found upon diagnostic imaging for other indications, these are counted as asymptomatic VTE. Treatment of these asymptomatic VTE will be determined by the attending physician.

Bleeding is categorized as major, clinically relevant non-major or minor bleeding according to Perinatal and Pediatric Subcommittee of the Scientific and Standardization Committee of the International Society on Thrombosis and Haemostasis criteria [27]. A bleeding is considered as major if it is a fatal bleeding, clinically overt bleeding associated with a decrease in hemoglobin of at least $3.1 \mathrm{mmol} / \mathrm{l}$ in a $24-\mathrm{h}$ period, bleeding that is retroperitoneal, pulmonary, intracranial, or otherwise involves the central nervous system; and bleeding that requires surgical intervention in an operating suite. A clinical relevant non-major bleeding is defined as an overt bleeding for which a blood product is administered and not directly attributable to the patient's underlying medical condition or a bleeding that requires medical or surgical intervention to restore hemostasis, not in an operating suite. All other overt bleedings or macroscopic evidence of bleeding are considered minor.

Burden of LMWH injections is estimated by a visual analogue scale (VAS) score and defined as a VAS score $>5$.

\section{Study population}

Children between 1 and 19 years of age with primary ALL, who are treated within the DCOG ALL-11 or subsequent study, are eligible for the TropicALL. Patients will be excluded if they are already using anticoagulant therapy upon screening, if they have active bleeding or a high risk for bleeding contraindicating anticoagulant therapy, including renal insufficiency (glomerular filtration rate $($ GFR $)<30 \mathrm{ml} / \mathrm{min} / 1.73 \mathrm{~m} 2$ ), hepatic disease associated with coagulopathy leading to a clinically relevant bleeding risk, stage 2 hypertension defined as blood pressure confirmed $>99$ th percentile $+5 \mathrm{mmHg}$, if they have a heparin allergy or a history of heparin-induced thrombocytopenia (HIT), or any other condition that, judged by the investigator, would place the patient at increased risk of harm due to participation. Thrombocytopenia is no exclusion criteria. However, LWMH should be temporarily interrupted, if platelet levels drop below $20 \times 10^{9} / \mathrm{L}$.

\section{ALL-11 study design}

All patients in ALL-11 are treated with a standardized treatment schedule. (Figs. 1, 2a and b.) All patients start with Induction IA and IB, after which patients are stratified according to their risk group. After risk group stratification, patients with Standard Risk ALL will be treated with cycle $M$, followed by cycle IV and maintenance therapy. Medium Risk group patients will be treated with cycle M, followed by Medium Risk Intensification and maintenance cycles. High Risk group patients will be treated with cycle $M$ if identified by minimal residual disease (MRD) or with High Risk blocks if identified earlier. After three High Risk blocks, patients will undergo assessment for allogeneic stem cell transplantation eligibility, or will continue High Risk blocks. PEGasparaginase is included in Induction IA (day 12, 26, 4; $1500 \mathrm{IU} / \mathrm{m} 2$ ) and, in case of continuous asparaginase treatment, IB (day 54, 68; individualized dose) and M (day 9, 23, 37, 51; individualized dose). In Medium Risk group patients (70\% of all patients), PEG-asparaginase is administered every 2 weeks for 14 times or 8 times during Intensification, depending on the randomization between standard and continuous asparaginase treatment. Both arms have the same number of total PEGasparaginase administrations.

\section{Intervention; LMWH regimen}

LMWH (nadroparin) will be given subcutaneously once daily, in a standardized prophylactic weight- and anti-Xa adjusted dose of $85 \mathrm{IU} / \mathrm{kg}$ with a maximum of $5700 \mathrm{IU} /$ day [28]. Previous studies have indicated that especially young children require higher dosages of LMWH. Hence, peak anti-Xa levels should be measured, 3 to 5 days after start of LMWH prophylaxis, with an aimed anti-Xa level of $0.3-0.4 \mathrm{IU} / \mathrm{ml}$ [29]. Lidocain/prilocain cream can be applied to the skin locally for anesthesia before each subcutaneous injection. If nadroparin is not tolerated, other LMWHs can be administered according 


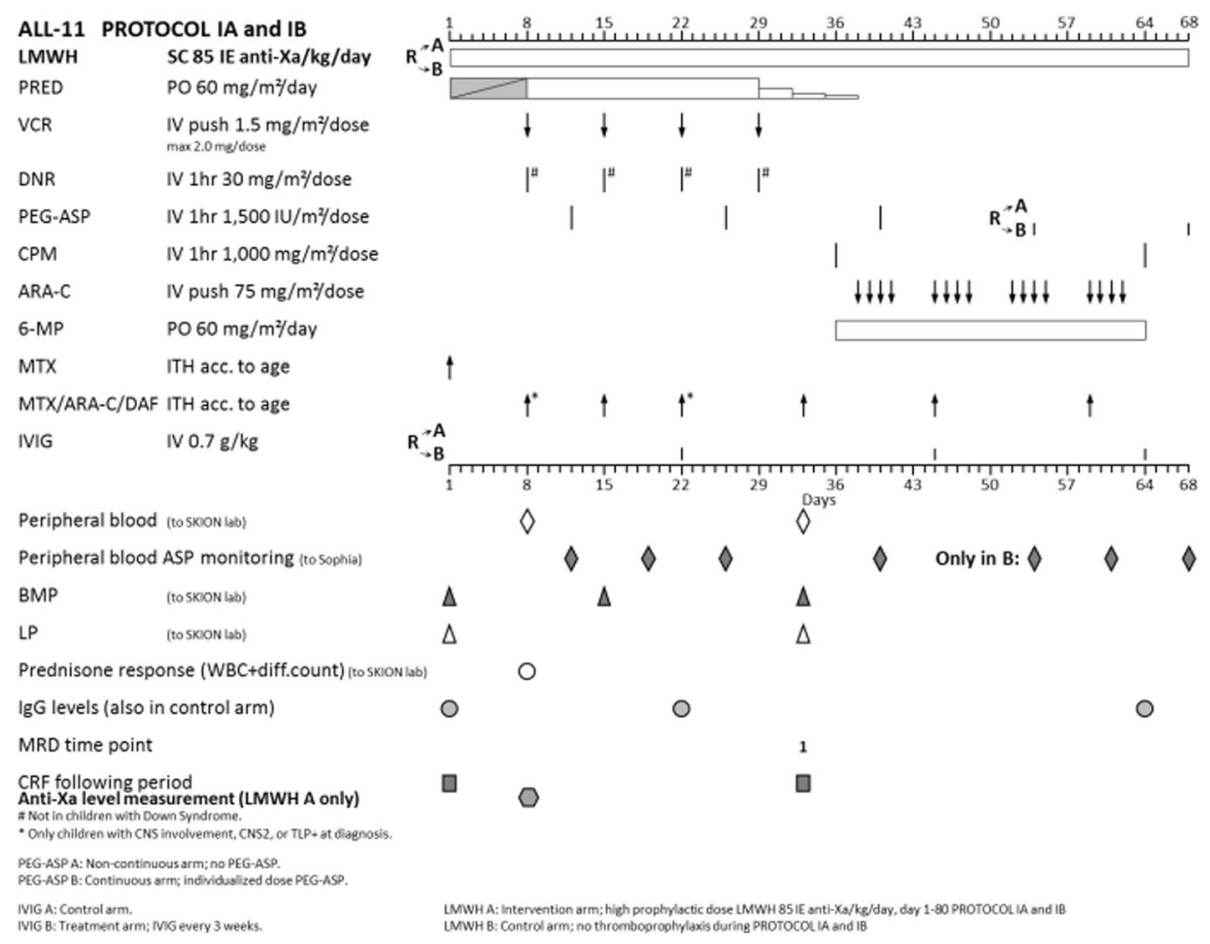

Fig. $1 \mathrm{LMWH}$ randomization in DCOG Protocol ALL 11, protocol 1A and 1B

to local availability (Table 1). A registration booklet and registration of unused drugs will be used to monitor treatment compliance. The booklets will also include questions on the burden of daily subcutaneous injections.

\section{Data and blood sample collection}

At the time of randomization for the TropicALL study and during the study, data will be collected from all patients, including baseline data, clinical risk factors for VTE, CVC characteristics, number of episodes of septicemia, asparaginase therapy and administration of other medications during ALL treatment.

Upon inclusion, one EDTA blood sample will be collected for evaluation of genetic thrombophilic mutations Factor V Leiden and Factor II mutation. Blood samples for coagulation assays, including antithrombin, fibrinogen, D-dimer, thrombin antithrombin complex (TAT) and thrombin generation, will be collected upon, during the induction cycle before the 2nd and 3rd asparaginase administration, and in Medium Risk group children in week 1 of Intensification and on the last day of PEGasparaginase therapy of Maintenance. All coagulation assays will be performed at the end of the study. Blood collection dates coincide with other set sample collection dates in ALL-11 or subsequent study and will not form an extra burden for patients.

\section{Sample size calculation}

Power calculations were based on an estimated symptomatic VTE incidence of $10 \%$ based on the previous DCOG ALL-10 study, and an estimated relative risk reduction (RRR) of $75 \%$ based on the abovementioned cohort studies with LMWH in childhood ALL [25, 26, 30, 31]. Using nQuery Advisor (7.0, 2007), it was calculated that 162 patients are required in each arm for a power of $80 \%$, using a two-sided test with significance level $\alpha=0.05$. Hence, 324 patients will be randomized in this study.

\section{Randomization}

Patients will be randomized to thromboprophylaxis with LMWH (arm A) or to no thromboprophylaxis ( $\operatorname{arm~B,}$ standard of care). Randomization will take place on day 11, which is the last day before the start of PEGasparaginase administration during the induction cycle. A register of all patients, who were eligible but not included in the study, will be kept and reason for refusal of participation will be recorded. Randomization of each patient will be performed by a randomization computer program at the DCOG trial office and will be stratified to ensure equal distribution of patients over study arms. Stratification will be done according to type of ALL (B-cell or T-cell) and study center. The procedure will also guarantee concealment of allocation by the treating physician. 


\section{a LMWH randomization in ALL-11 MR Intensification without anthracyclines}

ALL-11 MR Intensification + MR Maintenance part 1A without anthracyclines;

For TEL/AML1 without IKZF1 deletions and for Down syndrome without IKZF1 deletions

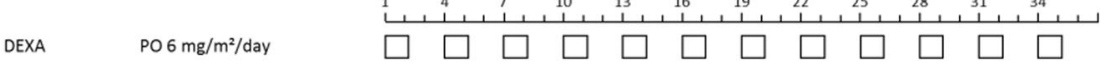

VCR IV push $2 \mathrm{mg} / \mathrm{m}^{2} /$ dose $\quad \downarrow \downarrow \downarrow \downarrow \downarrow \downarrow \downarrow \downarrow \downarrow \downarrow \downarrow \downarrow \downarrow \downarrow \downarrow \downarrow \downarrow$

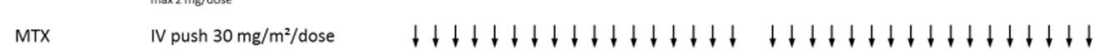

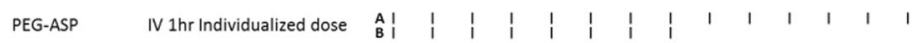

6-MP $\quad P O 50 \mathrm{mg} / \mathrm{m}^{2} /$ day

MTX/ARA-C/DAF ITH acc. to age

LMWH SC $85 \mathrm{IE}$ anti-Xa/kg/day

IVIG IV $0.7 \mathrm{~g} / \mathrm{kg}$

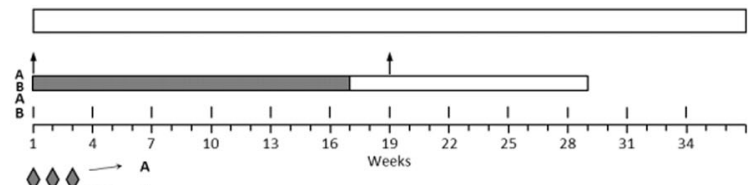

Peripheral blood ASP monitoring (to Sophla)

$\diamond \diamond \diamond \longrightarrow A$

BMP (toskionlab)

LP

IgG levels (also in control arm)

CRF following period

PEG-ASPA: Non-continuous arm; individualized dose PEG-ASP.
PEG-ASP B: Continuous arm: individualized dose PEG-ASP.

IVG A: Control arm.

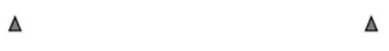

$\Delta \Delta$

b LMWH randomization in ALL-11 MR Intensification with anthracyclines

ALL-11 MR Intensification + MR Maintenance part 1B with anthracyclines

For IKZF1 deletions and for patients without IKZF deletions who have no TEL/AML1 and no Down syndrome

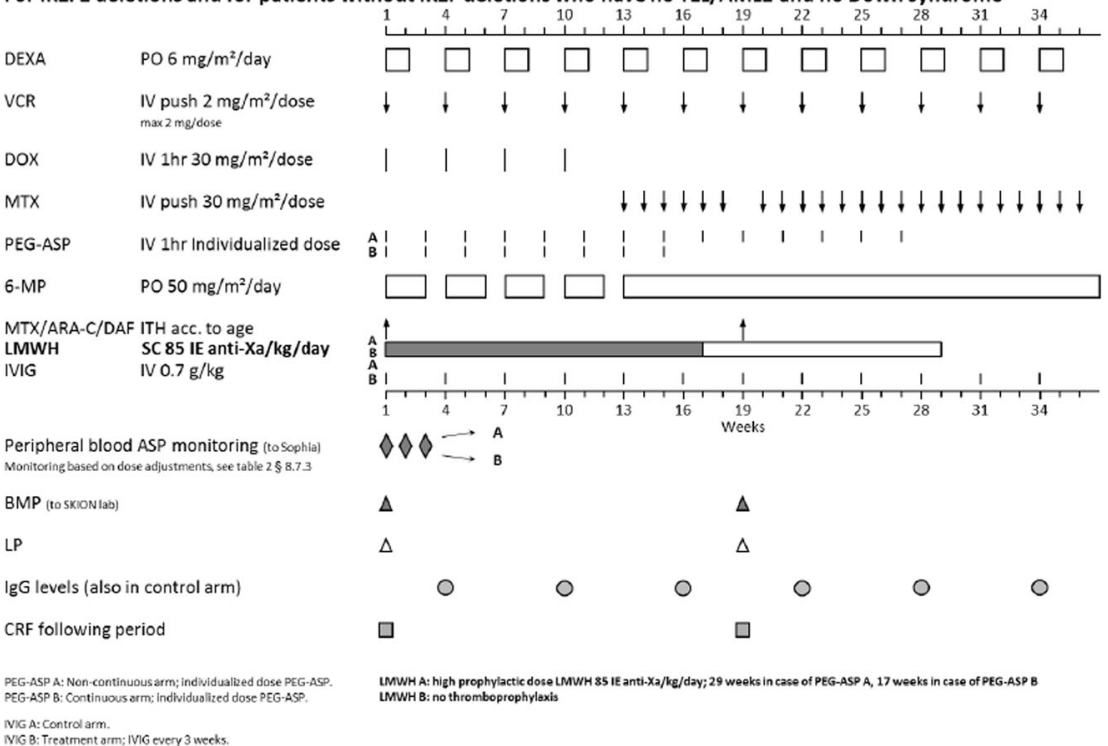

Fig. 2 a LMWH randomization in DCOG Protocol ALL 11, MR intensification, without anthracyclines. b LMWH randomization in DCOG Protocol ALL 11, MR intensification, with anthracyclines

Table 1 Alternative LMWH schedule

\begin{tabular}{ll}
\hline LMWH & Dose \\
\hline Enoxaparin & $1-18 \mathrm{y}: 1 \mathrm{mg} / \mathrm{kg}$ once daily s.c \\
Dalteparin & $65 \pm 21.5 \mathrm{IE} / \mathrm{kg}$ once daily s.c \\
Tinzaparin & $1-5 \mathrm{y}: 120 \mathrm{IE} / \mathrm{kg}$ once daily s.c. \\
& $5-10 \mathrm{y}: 100 \mathrm{IE} / \mathrm{kg}$ once daily s.c. \\
& $10-18 \mathrm{y}: 87.5 \mathrm{IE} / \mathrm{kg}$ once daily s.c.
\end{tabular}

Treatment arms in TropicALL

All patients randomized to arm A will receive LMWH from the day asparaginase is started, until 28 days (for PEG-asparaginase) after the end of asparaginase therapy, in all treatment cycles with asparaginase (Induction cycle, Intensification in Medium Risk protocol). In case PEG-asparaginase is replaced by Erwinia asparaginase, LMWH will be given until 7 days after the last administration of Erwinia asparaginase. Patients treated according the medium risk arm of the protocol will receive 
34 weeks (=238 days) of LMWH in the induction and intensification cycles, while patients treated according to the standard risk and high risk ALL-11 protocols will receive $\mathrm{LMWH}$ in the induction cycle only ( $=42$ days during and after asparaginase). Patients in arm $\mathrm{B}$, the standard of care arm, will not receive any form of thromboprophylaxis.

LMWH should be stopped $24 \mathrm{~h}$ before interventions, i.e. lumbar puncture or CVC insertion, and should be restarted after invasive procedures or surgical interventions within $24 \mathrm{~h}$, provided the clinical situation allows. Moreover, LMWH should be temporarily interrupted if platelet levels drop below $20 \times 10^{9} / \mathrm{L}$, and in case of major bleeding.

\section{Safety measures}

If major bleeding occurs, the following measures should be considered: (1) delay the next LMWH administration or discontinue treatment, (2) consider protamine sulfate administration, (3) consider usual treatment for bleeding, including blood transfusion, and/or fresh frozen plasma, (4) measure anti-Xa level of LMWH. If bleeding cannot be controlled, consider administration of recombinant factor VII (Novoseven ${ }^{\circ}$ ) or prothrombin complex concentrate.

\section{Reporting of symptomatic VTE events}

Patients are monitored for clinical symptoms of VTE during all treatment cycles of ALL-11 and a 3-month follow-up period. To establish systematic registration of symptomatic VTE, events must be prospectively centrally recorded on special VTE reporting forms in the CRF. VTE reporting forms include several specifications of the VTE to enable complete analysis of the event and its consequences for the patient and ALL treatment. For each symptomatic, objectively diagnosed VTE event in a study patient, a VTE reporting form should be completed and returned to the DCOG Office within 14 days after the event. Recurrent VTE should also be recorded.

Presence, replacement, removal or reinsertion of a CVC should be documented in the CRF, as well as the applied method of catheter flushing.

\section{Statistical analysis}

All data will be analyzed according to the intention-totreat principle. Descriptive data will be presented as mean with its corresponding standard deviation if normally distributed, and medians with ranges if data are skewed. Depending on the data distribution the Student's $t$ test or a Mann-Whitney U-test will be used to compare continuous variables between the treatment groups. Categorical variables will be analyzed using the Chisquare test.
Efficacy and safety of LMWH to prevent VTE will be analyzed by the difference between the incidence proportions and cumulative incidences of the primary efficacy and safety outcomes, symptomatic objectified VTE and major or clinically relevant non-major bleeding, respectively, observed from randomization up to 14 and 7 days after the last PEG asparaginase and Erwinia asparaginase, respectively. A two-sided test with significance level $\alpha=0.05$ will be used.

All bleeding events that occur during LMWH or within 2 days after cessation of LMWH will be included. Incidence proportions (number of children with outcome during the period divided by number of children at risk at the beginning of the period) and cumulative incidences will be estimated by a joint model to estimate event free survival for the primary safety outcome. To identify which risks factors are associated with time to VTE a joint model for longitudinal data and survival outcome will be used.

\section{Ethical considerations}

The study has been approved by the Ethics Committee of the Erasmus Medical Center in Rotterdam, the Netherlands, and the local Ethics Committee of each participating hospital. Patients can only be included in this study after obtaining written informed consent of both parents and children aged 12 and older. Verbal and written information on all parts of the study should be given.

We acknowledge, daily subcutaneous injections for the entire duration of asparaginase therapy could be a burden for patients. However, the high risk of VTE and potentially severe complications deriving from this VTE, transcend potential burden of dialy injections. Lidocain/ prilocain cream will be applied to diminish the pain of subcutaneous injections. To evaluate the burden, patients will receive a booklet with questions.

\section{Withdrawal of patients}

Children can discontinue their participation in the study at any time for any reason if they wish to do so without any consequences for their care, at their own request or at the request of their parents/legally acceptable representative. Furthermore, study medication can be stopped prematurely in case of serious adverse events, VTE requiring therapeutic anticoagulant treatment, or if, in the investigator's opinion, study medication should be stopped for any other reason.

\section{Discussion}

Despite the fact that thromboprophylaxis with LMWH has been proven to be safe in children for the prevention of VTE, there are no randomized studies that have investigated the use of thromboprophylaxis with $\mathrm{LMWH}$ during ALL treatment [32]. The TropicALL will be the 
first randomized controlled trial to investigate the safety and efficacy of LMWH as thromboprophylaxis in children treated for primary ALL. As a result of its incorporation in the DCOG ALL-11 and subsequent study protocols, optimal enrolment of patients is secured as well as implementation in all involved pediatric oncology centers. With the results of this study, we intend to establish (inter)national guidelines with recommendations for thromboprophylaxis during childhood ALL treatment.

\section{Abbreviations}

ALL: Acute lymphoblastic leukemia; AT: Antithrombin; CVC: Central venous catheter; CSVT: Cerebral sinovenous thrombosis; DCOG: Dutch Childhood Oncology Group; DVT: Deep venous thrombosis; FFP: Fresh frozen plasma; GFR: Glomerular filtration rate; HIT: Heparin-induced thrombocytopenia; LMWH: Low-molecular-weight heparin; PTS: Post thrombotic syndrome; RRR: Relative risk reduction; VTE: Venous thromboembolism

\section{Acknowledgements}

Not applicable.

\section{Funding}

This trial is funded by a project grant from The Netherlands Organisation for Health Research and Development, ZonMw Rational Pharmacotherapy Program no. 836011021

\section{Availability of data and materials}

The datasets used and/or analysed during the current study are available from the corresponding author on reasonable request.

\section{Authors' contributions}

$\mathrm{CO}, \mathrm{ML}, \mathrm{MW}, \mathrm{SM}$ and $\mathrm{BB}$ were involved in drafting the conception and design of the study. All other authors were involved in the final consensus process of the protocol and contributed significantly to the final version. IK $\mathrm{CO}, \mathrm{MW}$ drafted the manuscript and all other authors read, edited and approved the final manuscript.

\section{Competing interests}

The authors declare that they have no competing interests.

\section{Consent for publication}

Not applicable.

\section{Ethics approval and consent to participate}

The study has been centrally approved by the Ethics Committee of the Erasmus Medical Center in Rotterdam (MEC 2014-584), the Netherlands, and the local Ethics Committee of the Academic Medical Hospital (AMC), Amsterdam, the Netherlands.

Patients can only be included in this study after obtaining written informed consent of both parents and children aged 12 and older. Verbal and written information on all parts of the study should be given'.

\section{Author details}

'Department of Pediatric Hematology, Academic Medical Center, Amsterdam, The Netherlands. ${ }^{2}$ Department of Vascular Medicine, Academic Medical Center, Amsterdam, The Netherlands. ${ }^{3}$ Department of Hematology, Academic Medical Center, Amsterdam, The Netherlands. ${ }^{4}$ Department of Pediatric Oncology, Academic Medical Center, Amsterdam, The Netherlands. ${ }^{5}$ Department of Hematology/Oncology, VU Medical Center, Amsterdam, The Netherlands. ${ }^{6}$ Department of Hematology/Oncology, Wilhelmina Children's Hospital, Utrecht, The Netherlands. ${ }^{7}$ Department of Pediatric Hematology/ Oncology, Radboud University Medical Center, Nijmegen, The Netherlands. ${ }^{8}$ Princess Máxima Center for Pediatric Oncology, Utrecht, The Netherlands. ${ }^{9}$ Department of Pediatric Oncology/Hematology, Erasmus MC-Sophia Children's Hospital, Rotterdam, The Netherlands. ${ }^{10}$ Department of Pediatric Oncology, Beatrix Children's Hospital, University Medical Center Groningen, Groningen, the Netherlands.
Received: 24 November 2016 Accepted: 27 April 2017

Published online: 10 May 2017

\section{References}

1. Gatta G, Botta L, Rossi S, et al. Childhood cancer survival in Europe 19992007: results of EUROCARE-5-a population-based study. Lancet Oncol. 2014; 15:35-47.

2. Kaatsch P. Epidemiology of childhood cancer. Cancer Treat Rev. 2010;36: 277-85.

3. Pieters R, de Groot-Kruseman H, Van der Velden V, et al. Successful therapy reduction and intensification for childhood acute lymphoblastic leukemia based on minimal residual disease monitoring: study ALL10 from the Dutch Childhood Oncology Group. J Clin Oncol. 2016:34:2591-601.

4. Silverman LB, Gelber RD, Dalton VK, et al. Improved outcome for children with acute lymphoblastic leukemia: results of Dana-Farber Consortium Protocol 91-01. Blood. 2001:97:1211-8.

5. Coebergh JW, Pastore G, Gatta G, Corazziari I, Kamps W. Variation in survival of European children with acute lymphoblastic leukaemia, diagnosed in 1978-1992: the EUROCARE study. Eur J Cancer. 2001;37:687-94.

6. Cooper SL, Brown PA. Treatment of pediatric acute lymphoblastic leukemia. Pediatr Clin N Am. 2015;62:61-73

7. Athale UH, Chan AK. Thrombosis in children with acute lymphoblastic leukemia. Part 1. Epidemiology of thrombosis in children with acute lymphoblastic leukemia. Thromb Res. 2003;111:125-31.

8. Caruso V, lacoviello L, Di Castelnuovo A, et al. Thrombotic complications in childhood acute lymphoblastic leukemia: a metaanalysis of 17 prospective studies comprising 1752 pediatric patients. Blood. 2006;108:2216-22.

9. Payne JH, Vora AJ. Thrombosis and acute lymphoblastic leukaemia. Br J Haematol. 2007;138:430-45.

10. Mitchell LG, Andrew M, Hanna K, et al. Prophylactic Antithrombin Replacement in Kids with Acute Lymphoblastic Leukemia Treated with Asparaginase (PARKAA). A prospective cohort study determining the prevalence of thrombotic events in children with acute lymphoblastic leukemia and a central venous line who are treated with L-asparaginase: results of the Prophylactic Antithrombin Replacement in Kids with Acute Lymphoblastic Leukemia Treated with Asparaginase Group (PARKAA) study. Cancer. 2003;97:508-516

11. Ranta S, Tuckuviene R, Mäkipernaa A, et al. Cerebral sinus venous thromboses in children with acute lymphoblastic leukaemia - a multicentre study from the Nordic Society of Paediatric Haematology and Oncology. $\mathrm{Br}$ J Haematol. 2015;168:547-52.

12. deVeber $G$, Andrew M. Cerebral sinovenous thrombosis in children. N Engl J Med. 2001:345:417-23.

13. deVeber G, MacGregor D, Curtis R, Mayank S. Neurologic outcome in survivors of childhood arterial ischemic stroke and sinovenous thrombosis. Child Neurol. 2000;15:316-324.

14. Sébire G, Tabarki B, Saunders DE, et al. Cerebral venous sinus thrombosis in children: risk factors, presentation, diagnosis and outcome. Brain. 2005;128: 477-89.

15. Friefeld SJ, Westmacott R, Macgregor D, Deveber G. Predictors of quality of life in pediatric survivors of arterial ischemic stroke and cerebral sinovenous thrombosis. J Child Neurol. 2011;26:1186-92.

16. Kuhle S, Koloshuk B, Marzinotto V, et al. A cross-sectional study evaluating post-thrombotic syndrome in children. Thromb Res. 2003;111:227-33.

17. Manco-Johnson MJ. Postthrombotic syndrome in children. Acta Haematol. 2006;115:207-13.

18. Goldenberg NA, Donadini MP, Kahn SR, et al. Post-thrombotic syndrome in children: a systematic review of frequency of occurrence, validity of outcome measures, and prognostic factors. Haematologica. 2010;95:1952-9.

19. Barnes C, Newall F, Monagle P. Post-thrombotic syndrome. Arch Dis Child. 2002;86:212-4

20. Athale UH, Chan AKC. Thrombosis in children with acute lymphoblastic leukemia. Thromb Res. 2003;111:199-212.

21. Appel IM, van Kessel-Bakvis C, Stigter R, Pieters R. Influence of two different regimens of concomitant treatment with asparaginase and dexamethasone on hemostasis in childhood acute lymphoblastic leukemia. Leukemia. 2007; 21:2377-80

22. Bajzar L, Chan AK, Massicotte MP, Mitchell LG. Thrombosis in children with malignancy. Curr Opin Pediatr. 2006;18:1-9. 
23. Journeycake JM, Buchanan GR. Catheter-related deep venous thrombosis and other catheter complications in children with cancer. J Clin Oncol. 2006;24:4575-80.

24. Schoot RA, Kremer LCM, van de Wetering MD, van Ommen CH. Systemic treatments for the prevention of venous thrombo-embolic events in paediatric cancer patients with tunnelled central venous catheters. Cochrane Database Syst Rev. 2013;9:CD009160.

25. Elhasid R, Lanir N, Sharon R, et al. Prophylactic therapy with enoxaparin during L-asparaginase treatment in children with acute lymphoblastic leukemia. Blood Coagul Fibrinolysis. 2001;12:367-70.

26. Mitchell $L$, Lambers $M$, Flege $S$, et al. Validation of a predictive model for identifying an increased risk for thromboembolism in children with acute lymphoblastic leukemia: results of a multicenter cohort study. Blood. 2010; 115:4999-5004.

27. Mitchell LG, Male C. Outcome measures in interventional trials for prevention or treatment of venous thrombosis in the pediatric population. Semin Thromb Hemost. 2011;37:840-7.

28. Monagle P, Chan AKC, Goldenberg NA, et al. Antithrombotic therapy in neonates and children: Antithrombotic therapy and prevention of thrombosis, 9th ed: American college of chest physicians evidence-based clinical practice guidelines. Chest. 2012;141:e737S-801S.

29. Van Ommen $\mathrm{CH}$, van den Dool EJ, Peters M. Nadroparin therapy in pediatric patients with venous thromboembolic disease. J Pediatr Hematol Oncol. 2008:30:230-4.

30. Harlev D, Zaidman I, Sarig G, Ben Arush MW, Brenner B, Elhasid R. Prophylactic therapy with enoxaparin in children with acute lymphoblastic leukemia and inherited thrombophilia during L-asparaginase treatment. Thromb Res. 2010;126:93-7.

31. Meister B, Kropshofer G, Klein-Franke A, Strasak AM, Hager J, Streif W. Comparison of low-molecular-weight heparin and antithrombin versus antithrombin alone for the prevention of symptomatic venous thromboembolism in children with acute lymphoblastic leukemia. Pediatr Blood Cancer. 2008;50:298-303.

32. Monagle P, Chan AK, Goldenberg NA, et al. American College of Chest Physicians. Antithrombotic therapy in neonates and children: Antithrombotic therapy and prevention of thrombosis, 9th ed: American College of Chest Physicians Evidence-Based Clinical Practice Guidelines. Chest. 2012;141:e737S-801S.

\section{Submit your next manuscript to BioMed Central and we will help you at every step:}

- We accept pre-submission inquiries

- Our selector tool helps you to find the most relevant journal

- We provide round the clock customer support

- Convenient online submission

- Thorough peer review

- Inclusion in PubMed and all major indexing services

- Maximum visibility for your research

Submit your manuscript at www.biomedcentral.com/submit 